\title{
A semi-colloidal substrate for surface enhanced Raman scattering
}

Behzad Sardari

Meriç Özcan 


\title{
A semi-colloidal substrate for surface enhanced Raman scattering
}

\author{
Behzad Sardari and Meriç Özcan \\ Faculty of Engineering and Natural Science, Sabanci University, Istanbul - Turkey
}

\begin{abstract}
In this work, we utilize the electrolysis effect to prepare a semi-colloidal substrate for surface enhanced Raman spectroscopy (SERS) applications in which the nanoparticles created on the anode surface act as an active medium for SERS. The experiments carried out with copper $(\mathrm{Cu})$ as the electrode and Rhodamine B $(\mathrm{RhB})$ as the electrolyte. The measured enhancement factor $(E F)$ of the Raman peaks of $\mathrm{RhB}$ is more than five orders of magnitude. The proposed method has some key advantages: it is a very simple and low cost technique and also can be used in real time since it is a quite fast process.
\end{abstract}

Keywords: Surface enhanced Raman spectroscopy, semi-colloidal substrate, electrolysis, real time SERS substrate

\section{INTRODUCTION}

Preparing an appropriate surface enhanced Raman scattering substrate due to its importance in research laboratories and industries has been a central subject since the SERS was introduced in the mid-1970s. ${ }^{1-3}$ Since then different kinds of SERS substrates were developed like the assembly of colloidal nanoparticles ${ }^{4-10}$ in twodimensional and 3-dimensional configurations, patterned substrates ${ }^{11-15}$ fabricated by nano-scale fabrication techniques such as electron beam lithography (EBL), focused ion beam (FIB), scanning probe lithography etc. Generally, all these substrate building methods can be classified in two different categories; bottom-up methods, and top-down methods.

Although it is a time consuming process and appropriate for large scale production, synthesis of colloidal nanoparticles which is known as a bottom-up method, is straightforward. However potential aggregation of the nanoparticles over the substrate is a significant disadvantage of this method. On the other hand, patterning of the substrate which is known as a top-down method, provides means of adjusting the uniformity and the interparticle distance assuring the uniformity in enhancement factor, and absence of particles aggregation problem. Although it is repeatable, it is costly and time consuming, and much more complicated process which requires high-tech fabrication facilities.

Synthesis of nanoparticles by electrolysis which is the result of reduction and oxidation on the cathode and anode respectively, became popular recently. This is a bottom-up method, and considered for synthesis of colloidal nanoparticles for applications in the inkjet printing, SERS etc. ${ }^{16-18}$ Compared to most of the other synthesis methods of colloidal nanoparticles, this is a simple and economical method, and usually one needs a solution as an electrolyte that contains the ions of nanoparticles of interest. In particular, electrochemical method is one of the simplest way of producing copper oxide nanoparticles which is discussed in detail in the literature. ${ }^{19-21}$

In this work, we utilize the electrolysis effect to prepare a semi-colloidal substrate for SERS applications in which the nanoparticles created on the anode surface act as an active medium for SERS. The experiment carried out with copper $(\mathrm{Cu})$ as the electrode and Rhodamine $\mathrm{B}(\mathrm{RhB})$ as the electrolyte. The measured enhancement factor $(E F)$ of the Raman peaks of $\mathrm{RhB}$ is more than five orders of magnitude. The $E F$ versus time was also studied, which for 90 seconds electrolysis we obtained the maximum enhancement on the Raman peak of $1509 \mathrm{~cm}^{-1}$. In addition to the enhancement factor, the proposed method has some key advantages: it is a very simple and low cost technique and also can be used in real time since it is a quite fast process. ${ }^{22}$

Send correspondence to Meriç Özcan

Meriç Özcan: E-mail: meric@sabanciuniv.edu

Behzad Sardari: E-mail: behzadsardari@sabanciuniv.edu

Colloidal Nanoparticles for Biomedical Applications XII, edited by Marek Osiński, Wolfgang J. Parak, Xing-Jie Liang Proc. of SPIE Vol. 10078, 100780Y · (C) 2017 SPIE · CCC code: 1605-7422/17/\$18 · doi: 10.1117/12.2252240 


\section{EXPERIMENTAL SETUP}

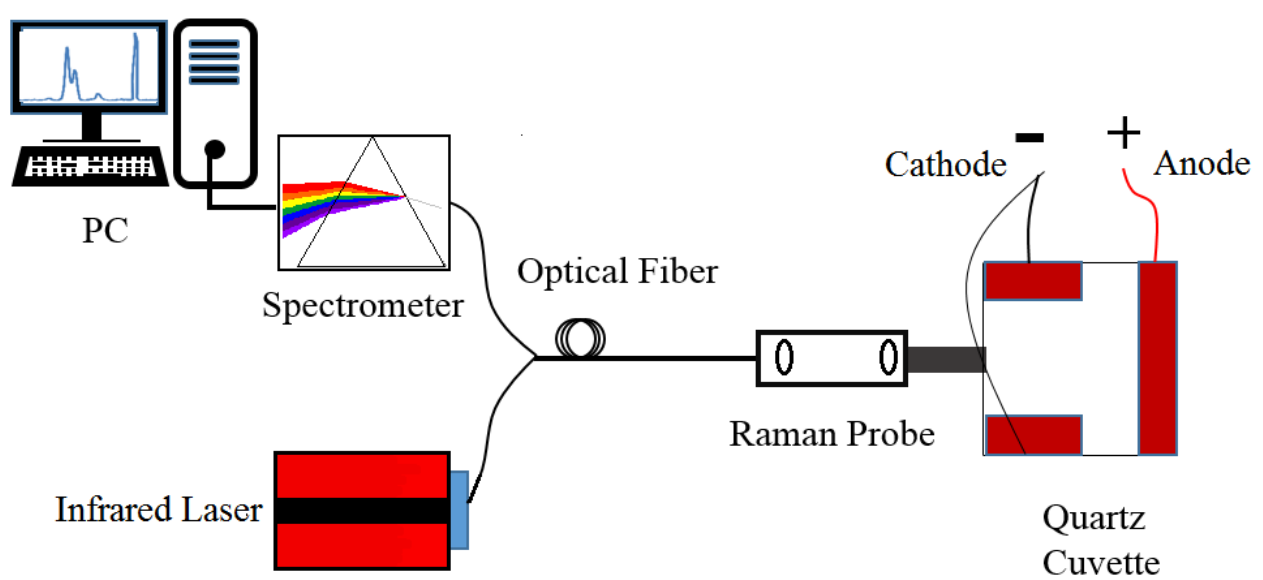

Figure 1. 2-D representation of the set-up (top-view) for recording Raman spectrum. The anode and cathode electrodes dimensions are $50 \mathrm{~mm} \times 8.5 \mathrm{~mm} \times 0.25 \mathrm{~mm}$ and $50 \mathrm{~mm} \times 5.5 \mathrm{~mm} \times 0.25 \mathrm{~mm}$ respectively. The Raman signal was recorded from the anode surface.

In order to perform the electrolysis experiment, 3 electrodes have been placed inside a $3 \mathrm{~mL}$ quartz cuvette in which two of them are parallel while the third one is perpendicular to these two electrodes, as illustrated in Fig. 1. The electrode from which the Raman signal was recorded is the anode while two other ones are used as the cathode. The applied voltage to the electrodes was $32 \mathrm{~V}$.

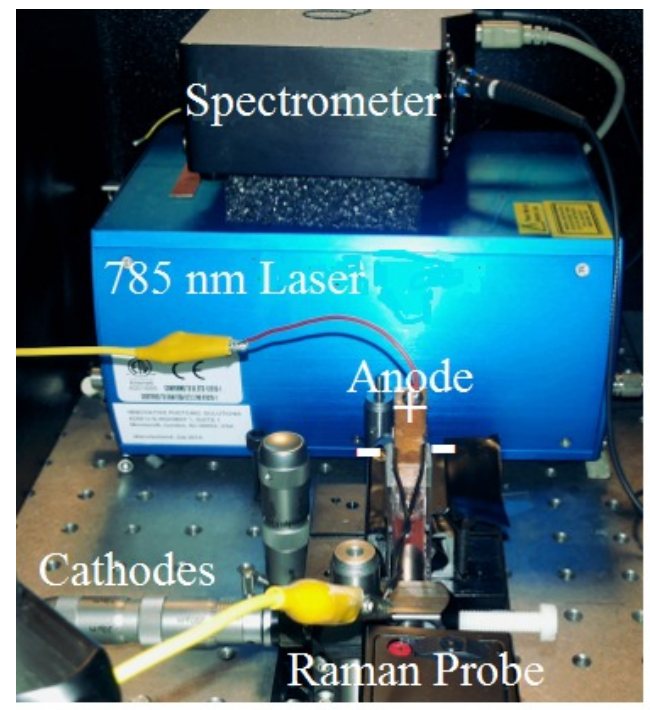

a

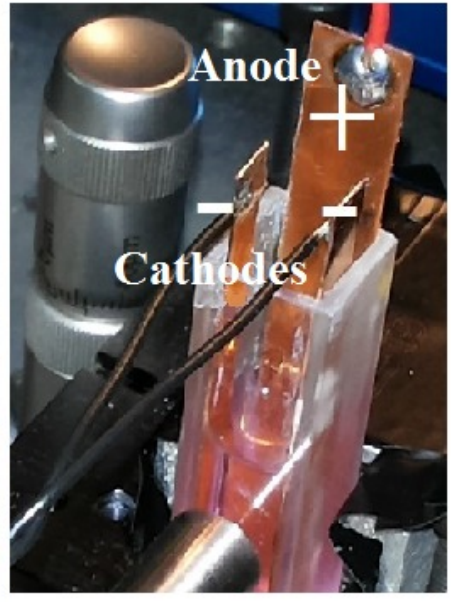

$\mathrm{b}$

Figure 2. a) A photograph of the whole set-up for recording Raman spectrum. The back-scattered light from the anode surface propagates through the Raman probe, then via an optical fiber it is transmitted to the spectrometer, b) a close up view of the quartz cuvette is shown.

Raman spectrum was recorded by a Raman probe made by InPhotonics Inc. And the other parts of the system are a QEpro spectrometer and an infrared laser source of $785 \mathrm{~nm}$, both made by Ocean Optics Inc. The quartz cuvette was located in front of the Raman probe and the back scattered Raman signal was recorded from the surface of the anode electrode as shown in Fig. 1. The photograph of the whole set-up is illustrated in Fig. 2. 


\section{EXPERIMENTAL RESULTS}

Initially, to investigate the SERS enhancement on the copper anode surface, we placed 3 copper electrodes inside a quartz cuvette of filled with $\mathrm{RhB}$ of $5 \mu \mathrm{M}$ then applied a $32 \mathrm{~V} \mathrm{DC}$ signal. In order to study the electrolysis effect on the Raman spectrum of RhB, the back scattered Raman spectrums from anode surface were recorded as a function of time. The enhancement factor $(E F)$ of the recorded Raman signals is calculated according to: ${ }^{10}$ $E F=\frac{I_{S E R S}}{I_{r e f}} \times \frac{C_{r e f}}{C_{S E R S}}$, where $I_{S E R S}, I_{r e f}, C_{r e f}$ and $C_{S E R S}$ correspond to recorded Raman signal intensity from the SERS substrate, intensity of the reference Raman signal, concentration of the reference sample, and concentration of the SERS sample respectively. In order to obtain the optimum required time for electrolysis -to get maximum $E F$ - we recorded the Raman spectrum at various electrolysis times. The recorded spectrums from $\mathrm{Cu}$ electrode (anode) surface and also the variation of $E F$ for the Raman peak of $1509 \mathrm{~cm}^{-1}$ are shown in Fig. 3. As it is clear from this figure, even for 30 seconds electrolysis, the anode surface functions as a SERS active substrate as the Raman $E F$ reaches above four orders of magnitude. The maximum $E F$ (roughly $1.5 \times 10^{5}$ ) occurred at 90 seconds electrolysis then it started to reduce gradually for further electrolysis which after ten minutes of electrolysis time the $E F$ decreased to almost fifty percent of the maximum value. These results are discussed in detail in the next section.

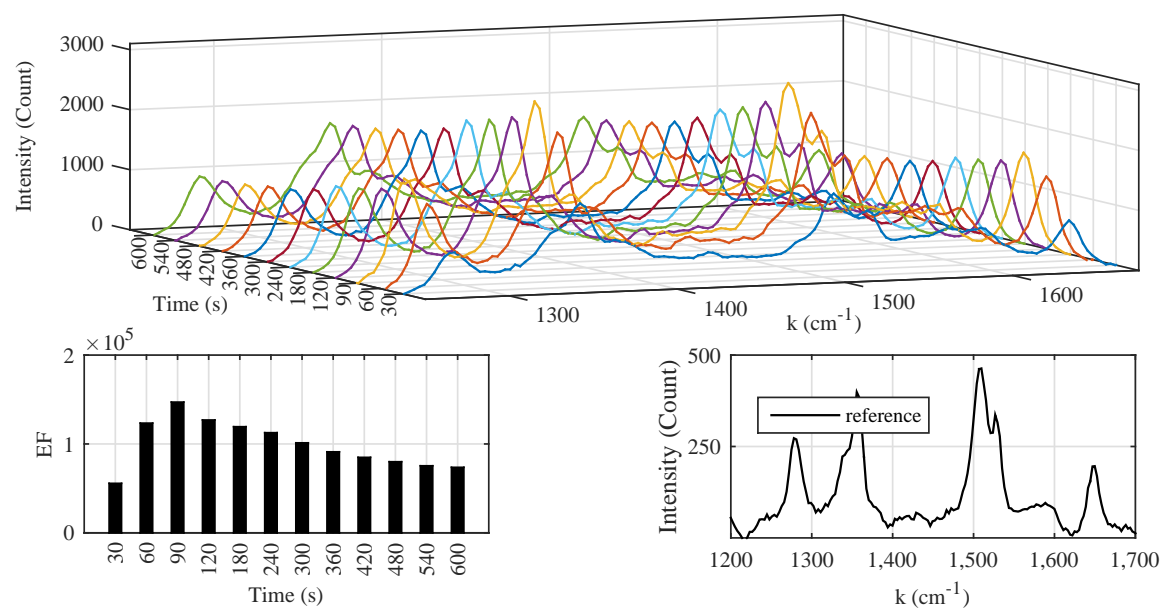

Figure 3. $E F$ of $5 \mu M \mathrm{RhB}$ variation for different electrolysis times. For electrolysis time of 90 seconds the $E F$ for the Raman peak of $1509 \mathrm{~cm}^{-1}$ reaches the maximum value of above 5 orders of magnitude. Reference spectrum was recorded from RhB of concentration of $100 \mathrm{mM}$ in a second.

The substrate $E F$ uniformity for the Raman peak of $1509 \mathrm{~cm}^{-1}$ was quantified by calculating the $E F$ on 6 random spots through a roughly $1 \mathrm{~cm}^{2}$ area of the $\mathrm{Cu}$ anode surface after 90 seconds electrolysis. The $E F$ variation through these spots had a relative standard deviation (RSD) of roughly 11.7 percent. The spectrums of these spots and also the $E F$ are shown in Fig. 4. A uniform $E F$ was expected from the surface due to uniform current density on the surface which leads to uniform nanoparticles production on the substrate.

\section{SURFACE ANALYSIS AND SIMULATION RESULTS}

In order to have a better understanding of the Raman enhancement on the copper anode surface, we recorded the scanning electron microscopy (SEM) images of the substrates. The recorded SEM images show the production of copper oxide nanoparticles of the size of a few hundred nanometers. As it is shown in Fig. 5, at ninety seconds electrolysis the nanocubes size ranges from $30 \mathrm{~nm}$ to $150 \mathrm{~nm}$ with average size of $100 \mathrm{~nm}$. However, at five minutes electrolysis, the nanocubes size ranges from $50 \mathrm{~nm}$ to $420 \mathrm{~nm}$ with average size of $300 \mathrm{~nm}$.

The production of copper oxide nanoparticles on the anode surface can be explained by the tendency of copper to oxidation. Since the copper anode try to donate electrons and to form $C u^{2+}\left(C u \rightarrow C u^{2+}+2 e^{-}\right)$, there are $\mathrm{Cu}^{2+}$ cations on the anode surface and in the electrolyte. Some of these cations contribute in the reduction 

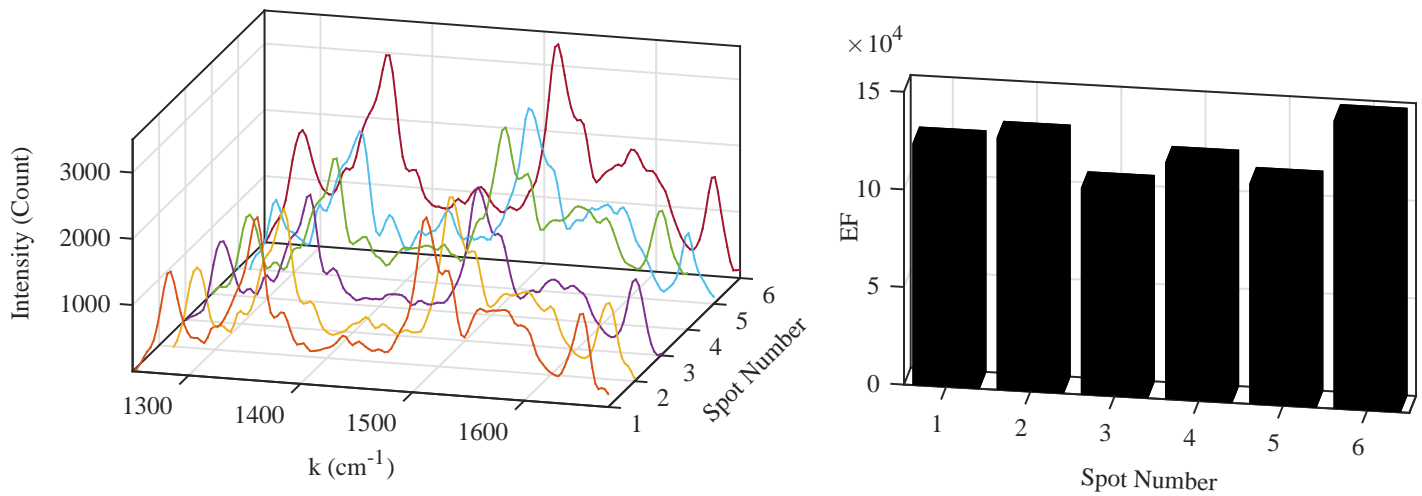

Figure 4. The recorded Raman spectrums from 6 random spots on the $\mathrm{Cu}$ substrate (left), comparison of $E F$ through these spots (right). Calculated RSD of the $E F$ variation for the Raman peak of $1509 \mathrm{~cm}^{-1}$ is 11.7 percent. The recorded spectrums and calculated results were done for 90 seconds electrolysis time and RhB of concentration of $5 \mu M$.

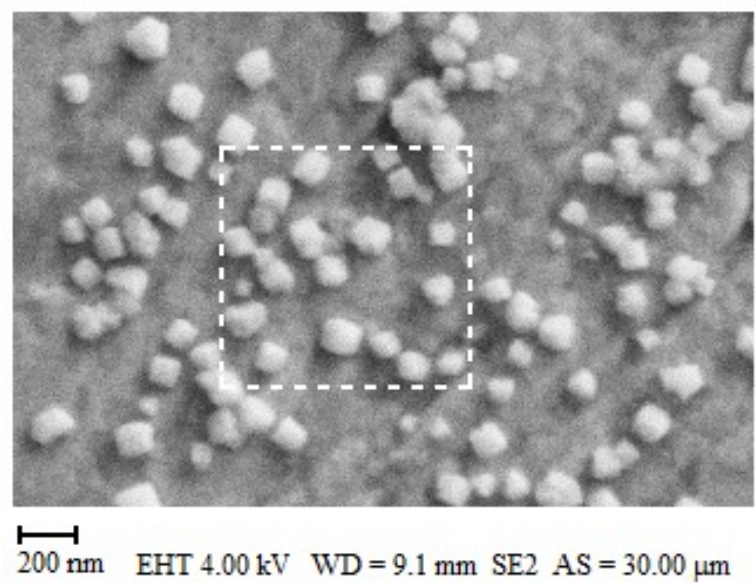

a

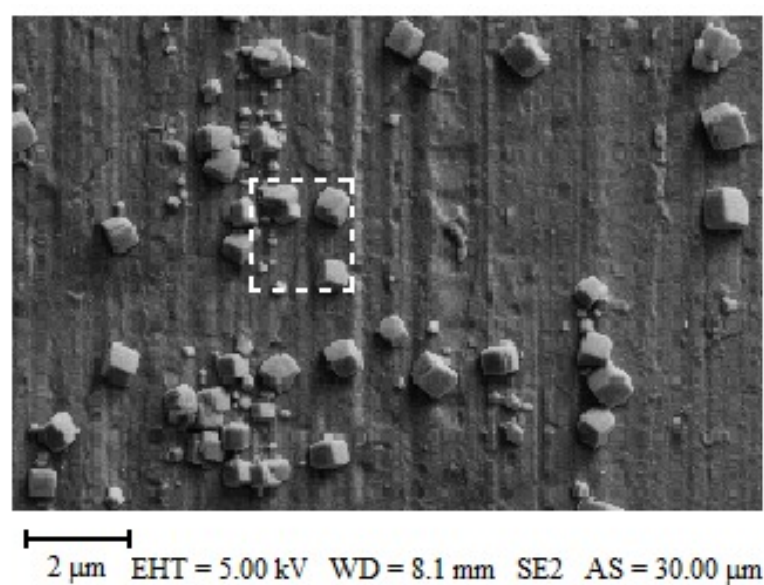

$\mathrm{b}$

Figure 5. SEM images from anode surface of copper after a) 90 seconds electrolysis, b) 5 minutes electrolysis. In both cases the containers were filled with $\mathrm{RhB}$ of concentration of $5 \mu \mathrm{M}$.

process to produce $\mathrm{Cu}$ nanoparticles $\left(\mathrm{Cu}_{2+}+2 e_{-} \rightarrow \mathrm{Cu}\right)$ and some others contribute in the copper oxidation process $\left(\mathrm{Cu}_{2} \mathrm{O}, \mathrm{CuO}\right.$, etc). In this experiment the growth of the copper oxide nanoparticles is a fast process that in a few minutes the nanoparticles size reaches to about $400 \mathrm{~nm}$ (see Fig. 5), and these particles are responsible for enhancing local electric field for SERS applications.

Later, we used FDTD technique to simulate the surface Plasmon resonance (SPR) wavelength for different nanoparticle sizes on the copper surface to explain the nanoparticle size effect on the EF. For this purpose, we selected a realistic collection of particles as shown with the dashed squares in Fig. 5. For the ninety second run simulations, we selected the $1 \mu \mathrm{m} \times 1 \mu \mathrm{m}$ area with nanocubes size ranging from $80 \mathrm{~nm}$ up to $150 \mathrm{~nm}$, while for five minutes run simulation, we selected the $2 \mu \mathrm{m} \times 2 \mu \mathrm{m}$ area with nanocubes size ranging from $90 \mathrm{~nm}$ up to $350 \mathrm{~nm}$. The nanoparticles distribution and simulation results for both cases are shown in Fig. 6. As it is clear from this figure, the extinction cross section for the case of ninety seconds electrolysis has the resonance wavelength of $790 \mathrm{~nm}$, while for the case of five minutes electrolysis this resonance wavelength is $1015 \mathrm{~nm}$. This difference in resonance wavelengths for two cases affects the enhancement factor. Since the substrate is excited with $785 \mathrm{~nm}$ laser source, the substrate with resonance wavelength of closer to the excitation wavelength will have better enhancement factor on the SERS. In a nutshell, since, increasing the electrolysis time leads to increment in nanocubes size, and consequently the red shift in resonance wavelength, the reduction in SERS EF is justifiable. 


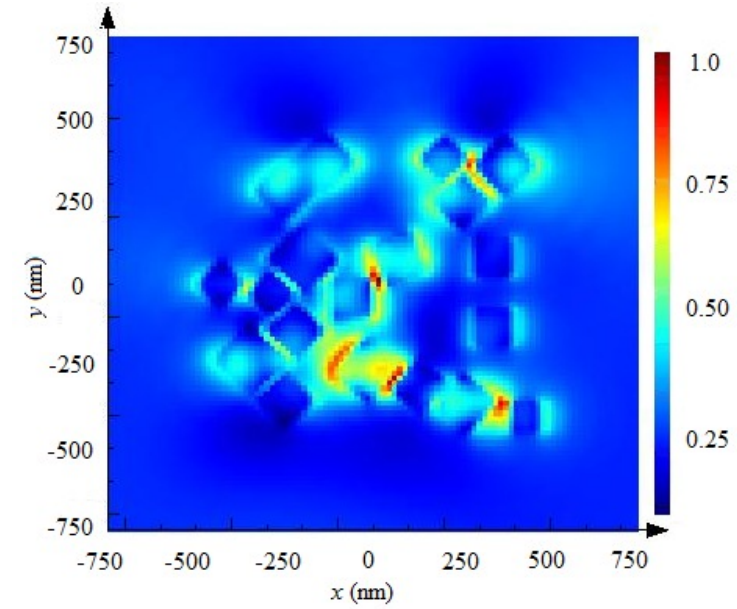

a

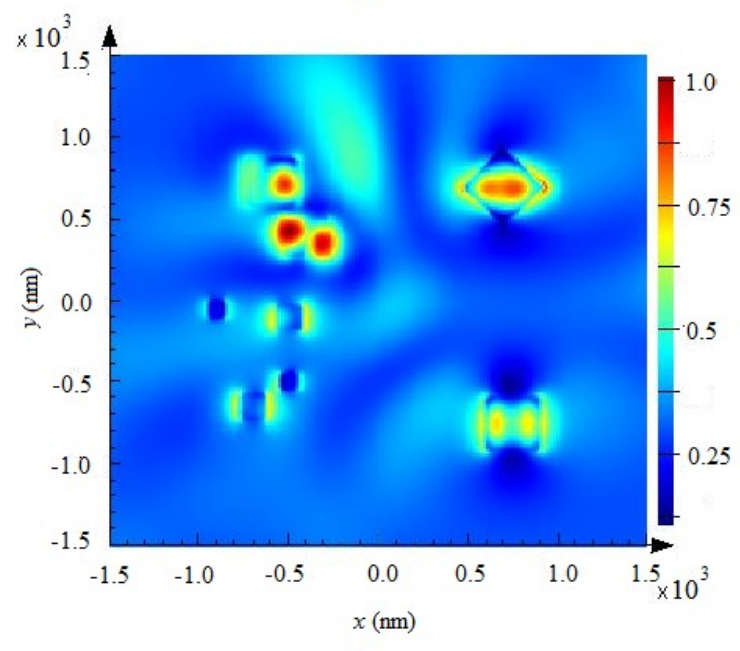

$\mathrm{c}$

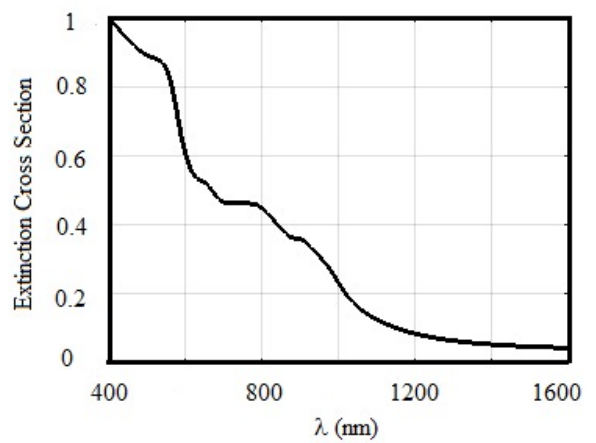

$\mathrm{b}$

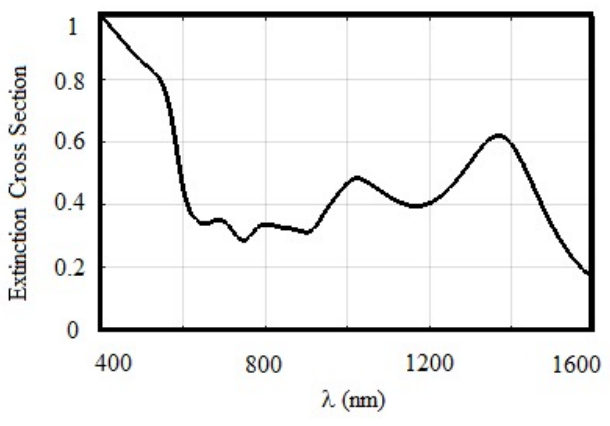

$\mathrm{d}$

Figure 6. a) electric field amplitude distribution at $50 \mathrm{~nm}$ above the surface at SPR resonance of $790 \mathrm{~nm}$ for the case of ninety seconds electrolysis, b) the extinction cross section of this assembly of nanocubes as a function of wavelength, c) electric field amplitude distribution at $50 \mathrm{~nm}$ above the surface at SPR resonance of $1015 \mathrm{~nm}$ for the case of five minutes electrolysis, and d) the extinction cross section of this assembly of nanocubes as a function of wavelength. In both cases the substrate is copper.

\section{CONCLUSION}

In conclusion, in this work we prepared a semi-colloidal SERS active substrate by utilizing the electrolysis effect on the copper anode surface. The produced copper oxide nanoparticles on the anode surface in matter of minutes acted as an active medium for SERS. Rhodamine B of concentration of $5 \mu M$ used as the electrolyte and its Raman signal at different electrolysis time recorded to obtain the maximum $E F$. In the carried out experiments we measured more than five orders of magnitude enhancement on the Raman peak of $1509 \mathrm{~cm}^{-1}$ for 90 seconds electrolysis. The $E F$ started to reduce gradually for further electrolysis which after 10 minutes, this value decreased to almost 50 percent of the maximum value. The proposed method has some key advantages: it is a very simple and low cost technique and also can be used in real time since it is a quite fast process. 


\section{ACKNOWLEDGMENTS}

Finally, we like to thank to The Scientific and Technological Research Council of Turkey (TÜBİTAK, project number: 113F357) for funding this project.

\section{REFERENCES}

[1] Martin Fleischmann, Patrick J Hendra, and AJ McQuillan. Raman spectra of pyridine adsorbed at a silver electrode. Chemical Physics Letters, 26(2):163-166, 1974.

[2] David L Jeanmaire and Richard P Van Duyne. Surface Raman spectroelectrochemistry: Part i. heterocyclic, aromatic, and aliphatic amines adsorbed on the anodized silver electrode. Journal of Electroanalytical Chemistry and Interfacial Electrochemistry, 84(1):1-20, 1977.

[3] M Grant Albrecht and J Alan Creighton. Anomalously intense Raman spectra of pyridine at a silver electrode. Journal of the American Chemical Society, 99(15):5215-5217, 1977.

[4] Bhavya Sharma, M Fernanda Cardinal, Samuel L Kleinman, Nathan G Greeneltch, Renee R Frontiera, Martin G Blaber, George C Schatz, and Richard P Van Duyne. High-performance SERS substrates: Advances and challenges. MRS Bulletin, 38(08):615-624, 2013.

[5] Yuliang Wang and Younan Xia. Bottom-up and top-down approaches to the synthesis of monodispersed spherical colloids of low melting-point metals. Nano Letters, 4(10):2047-2050, 2004.

[6] Xiaoshuang Shen, Liyong Chen, Dehui Li, Liangfang Zhu, Hong Wang, Cuicui Liu, Yong Wang, Qihua Xiong, and Hongyu Chen. Assembly of colloidal nanoparticles directed by the microstructures of polycrystalline ice. ACS Nano, 5(10):8426-8433, 2011.

[7] Ales Panacek, Libor Kvitek, Robert Prucek, Milan Kolar, Renata Vecerova, Nadezda Pizurova, Virender K Sharma, Tat'jana Nevecna, and Radek Zboril. Silver colloid nanoparticles: synthesis, characterization, and their antibacterial activity. The Journal of Physical Chemistry B, 110(33):16248-16253, 2006.

[8] Taeghwan Hyeon. Chemical synthesis of magnetic nanoparticles. Chemical Communications, (8):927-934, 2003.

[9] Fei Hu, Haiyang Lin, Zhaoshun Zhang, Fan Liao, Mingwang Shao, Yeshayahu Lifshitz, and Shuit-Tong Lee. Smart liquid SERS substrates based on Fe3O4/Au nanoparticles with reversibly tunable enhancement factor for practical quantitative detection. Scientific Reports, 4, 2014.

[10] Kihoon Kim, Hye Soo Han, Inhee Choi, Chiwon Lee, SoonGweon Hong, Sang-Hee Suh, Luke P Lee, and Taewook Kang. Interfacial liquid-state surface-enhanced Raman spectroscopy. Nature Communications, 4, 2013.

[11] Werayut Srituravanich, Nicholas Fang, Cheng Sun, Qi Luo, and Xiang Zhang. Plasmonic nanolithography. Nano Letters, 4(6):1085-1088, 2004.

[12] Hua Zhang, Sung-Wook Chung, and Chad A Mirkin. Fabrication of sub-50-nm solid-state nanostructures on the basis of dip-pen nanolithography. Nano Letters, 3(1):43-45, 2003.

[13] GM Wallraff and WD Hinsberg. Lithographic imaging techniques for the formation of nanoscopic features. Chemical Reviews, 99(7):1801-1822, 1999.

[14] ES Snow, PM Campbell, and FK Perkins. High speed patterning of a metal silicide using scanned probe lithography. Applied Physics Letters, 75(10):1476-1478, 1999.

[15] Jon Orloff, LW Swanson, and Mark Utlaut. Fundamental limits to imaging resolution for focused ion beams. Journal of Vacuum Science \&3 Technology B, 14(6):3759-3763, 1996.

[16] Jin Min Cheon, Jin Ha Lee, Yongsul Song, and Jongryoul Kim. Synthesis of Ag nanoparticles using an electrolysis method and application to inkjet printing. Colloids and Surfaces A: Physicochemical and Engineering Aspects, 389(1):175-179, 2011.

[17] Jinmin Cheon, Jinha Lee, and Jongryoul Kim. Inkjet printing using copper nanoparticles synthesized by electrolysis. Thin Solid Films, 520(7):2639-2643, 2012. 
[18] Xiao-Feng Tang, Zhen-Guo Yang, and Wei-Jiang Wang. A simple way of preparing high-concentration and high-purity nano copper colloid for conductive ink in inkjet printing technology. Colloids and Surfaces A: Physicochemical and Engineering Aspects, 360(1):99-104, 2010.

[19] Gao-Qing Yuan, Huan-Feng Jiang, Chang Lin, and Shi-Jun Liao. Shape-and size-controlled electrochemical synthesis of cupric oxide nanocrystals. Journal of Crystal Growth, 303(2):400-406, 2007.

[20] Qiaobao Zhang, Kaili Zhang, Daguo Xu, Guangcheng Yang, Hui Huang, Fude Nie, Chenmin Liu, and Shihe Yang. $\mathrm{CuO}$ nanostructures: synthesis, characterization, growth mechanisms, fundamental properties, and applications. Progress in Materials Science, 60:208-337, 2014.

[21] Minwei Xu, Fei Wang, Bingjun Ding, Xiaoping Song, and Jixiang Fang. Electrochemical synthesis of leaf-like $\mathrm{CuO}$ mesocrystals and their lithium storage properties. RSC Advances, 2(6):2240-2243, 2012.

[22] Behzad Sardari and Meriç Özcan. Real-time and tunable substrate for surface enhanced Raman spectroscopy by synthesis of copper oxide nanoparticles via electrolysis. Submitted to Scientific Reports, 2017. 